\title{
Erratum: $\mathrm{NbS}_{3}$ : A unique quasi-one-dimensional conductor with three charge density wave transitions [Phys. Rev. B 95, 035110 (2017)]
}

S. G. Zybtsev, V. Ya. Pokrovski, V. F. Nasretdinova, S. V. Zaitsev-Zotov, V. V. Pavlovskiy, A. B. Odobesco, Woei Wu Pai, M.-W. Chu, Y. G. Lin, E. Zupanič, H. J. P. van Midden, S. Šturm, E. Tchernychova, A. Prodan, J. C. Bennett,

I. R. Mukhamedshin, O. V. Chernysheva, A. P. Menushenkov, V. B. Loginov, B. A. Loginov,

A. N. Titov, and M. Abdel-Hafiez

(Received 9 December 2018; published 4 January 2019)

DOI: 10.1103/PhysRevB.99.039901

In Fig. 8 of the original paper, the arrow " $T_{\mathrm{P} 0}$ " erroneously indicates an irreversible drop of resistance at higher temperatures. The correct value of $T_{\mathrm{P} 0}$ is $450-475 \mathrm{~K}$. A small feature around $T_{\mathrm{P} 0}$ is indicated by an arrow in the new Fig. 1, which should replace Fig. 8 of the original paper. A similar curve for another sample is added to show the $T_{\mathrm{P} 1}$ and $T_{\mathrm{P} 0}$ transitions.

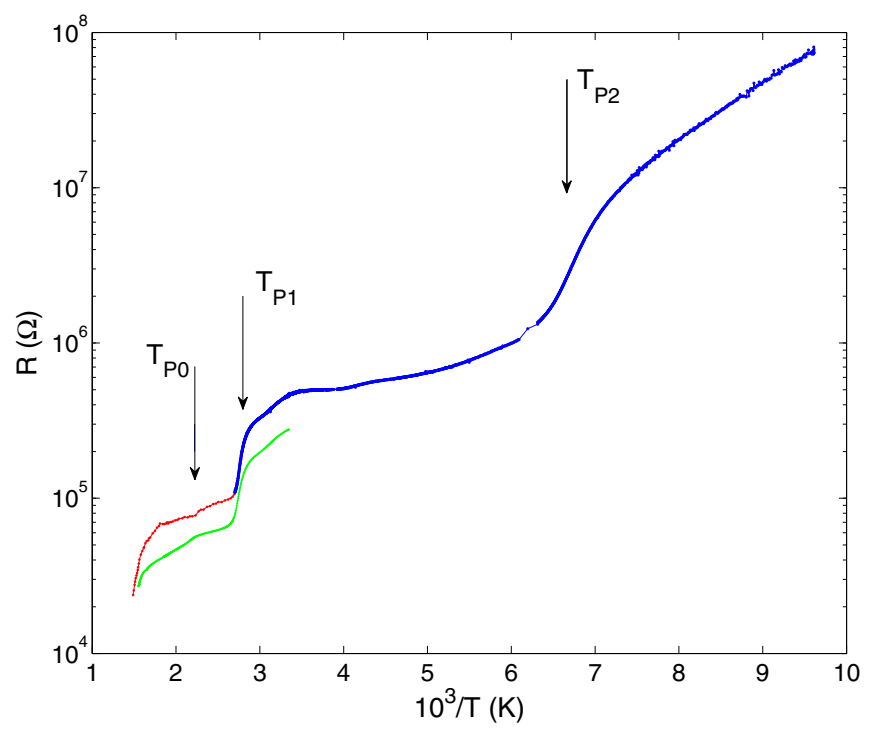

FIG. 1. The upper curve repeats Fig. 8 from our paper with $T_{\mathrm{P} 0}$ correctly indicated: A wide-range temperature dependence of NbS 3 -II resistance. Data from two whiskers $\left(100 \times 0.09 \mu \mathrm{m}^{2}\right.$ - high $T, 126 \times 0.06 \mu \mathrm{m}^{2}$-lower $\left.T\right)$ are combined into a single graph. The hightemperature points were obtained during a heating cycle in an Ar flow. The lower green curve shows a similar dependence recently obtained for another sample (160- $\mu \mathrm{m}$ length). 